\title{
Semantics and Reasoning Algorithms for a Faithful Integration of Description Logics and Rules
}

\author{
Boris Motik \\ University of Oxford, UK
}

\begin{abstract}
Description logics (DLs) and rule-based systems are two prominent families of knowledge representation formalisms. While DLs are focused on describing and reasoning about conceptual knowledge, rules are focused on answering queries about facts in the knowledge base. So far, research on DLs has been largely isolated from the research on rules. With the advent of the Semantic Web, however, it became apparent that neither formalism alone can cover all the practical use cases. The integration between DLs and rules, however, is technically challenging due to significant differences in the underlying semantic assumptions. In particular, DLs are based on standard first-order semantics and openworld assumption, whereas rules typically employ closed-world semantics based on a variant of circumscription.

In my talk, I shall present an overview of the benefits of integrating DLs and rules in a coherent semantic framework, and shall discuss the main challenges in achieving a tight integration. I shall present an overview of the approaches currently discussed in literature. Finally, I shall present in more detail the approach that is based on the nonmonotonic logic MKNF by Lifschitz. This approach is tight in the sense that DLs and rules are given a common model-theoretic semantics. Furthermore, it is faithful in the sense that it is compatible with the original semantics of both DLs and rules. Finally, it is expressive and can naturally capture many of the existing proposals. I shall discuss the definitions of the semantics of the hybrid formalism, present a decision procedure for a particular decidable fragment, and discuss the complexity bounds of reasoning.
\end{abstract}

